\title{
Open Heavy Flavor and Quarkonia Results at RHIC
}

\author{
Rachid Nouicer ${ }^{1,2, a}$ \\ ${ }^{1}$ Physics Department, Brookhaven National Laboratory, Upton, New York 11973, United States \\ ${ }^{2}$ RIKEN-BNL Research Center, Brookhaven National Laboratory, Upton, New York 11973, United States
}

\begin{abstract}
RHIC experiments carry out a comprehensive physics program which studies open heavy flavor and quarkonium production in relativistic heavy-ion collisions. The discovery at RHIC of large high- $p_{\mathrm{T}}$ suppression and flow of electrons from heavy quarks flavors have altered our view of the hot and dense matter formed in central $\mathrm{Au}+\mathrm{Au}$ collisions at $\sqrt{s_{N N}}=200 \mathrm{GeV}$. These results suggest a large energy loss and flow of heavy quarks in the hot, dense matter. In recent years, the RHIC experiments upgraded the detectors; (1) PHENIX Collaboration installed silicon vertex tracker (VTX) at mid-rapidity region and forward silicon vertex tracker (FVTX) at the forward rapidity region, and (2) STAR Collaboration installed the heavy flavor tracker (HFT) and the muon telescope detector (MTD) both at the mid-rapidity region. With these new upgrades, both experiments have collected large data samples. These new detectors enhance the capability of heavy flavor measurements via precision tracking. The PHENIX experiments established measurements of $\psi(1 S)$ and $\psi(2 S)$ production as a function of system size, $p+p$, $p+\mathrm{Al}, p+\mathrm{Au}$, and ${ }^{3} \mathrm{He}+\mathrm{Au}$ collisions at $\sqrt{s_{N N}}=200 \mathrm{GeV}$. In $\mathrm{p} /{ }^{3} \mathrm{He}+\mathrm{A}$ collisions at forward rapidity, we observe no difference in the $\psi(2 S) / \psi(1 S)$ ratio relative to $p+p$ collisions. At backward rapidity, where the comoving particle density is higher, we find that the $\psi(2 S)$ is preferentially suppressed by a factor of two. STAR Collaboration presents the first $J / \psi$ and $\Upsilon$ measurements in the di-muon decay channel in $\mathrm{Au}+\mathrm{Au}$ collisions at $\sqrt{s_{N N}}=200 \mathrm{GeV}$ at mid-rapidity at RHIC. We observe clear $J / \psi R_{\mathrm{AA}}$ suppression and qualitatively well described by transport models simultaneously accounting for dissociation and regeneration processes.
\end{abstract}

\section{Physics Motivation}

At the heart of each atom, there is a nucleus consisting of distinct nucleons (protons and neutrons). In turn, these nucleons consist of quarks bound together by the strong interaction, mediated by the exchange of gluons. Quantum Chromodynamics (QCD) is considered the fundamental theory for such strong interactions. According to QCD, at ordinary temperatures or densities this force simply confines the quarks into composite hadrons. However, when the temperature reaches the QCD energy scale ( $\mathrm{T}$ of the order of $10^{12}$ Kelvin) or its density rises to the point where the average inter-quark separation is less than $1 \mathrm{fm}$ (quark chemical potential $\mu_{\mathrm{q}}$ around $400 \mathrm{MeV}$ ), hadronic matter under extremely dense and hot conditions undergoes a phase transition to form a Quark Gluon Plasma (QGP) in which quarks and gluons no longer are confined to the size of a hadron [1,2]. Knowing the exact

ae-mail: rachid.nouicer@bnl.gov 


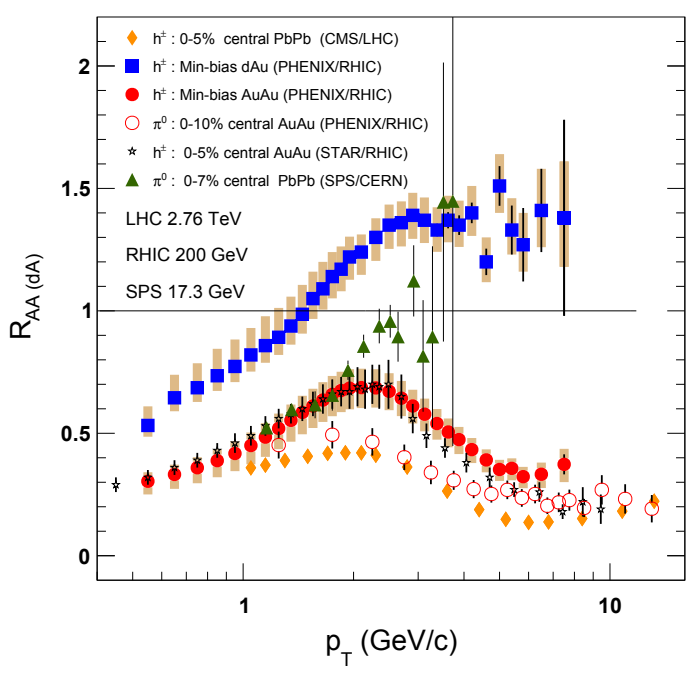

Figure 1. The evolution of the nuclear modification factor with center-of-mass energy, from the SPS to RHIC and then to the LHC. The error bars correspond to the statistical errors. For clarity, the systematic errors are shown as vertical bands. For more details see Ref. [4].

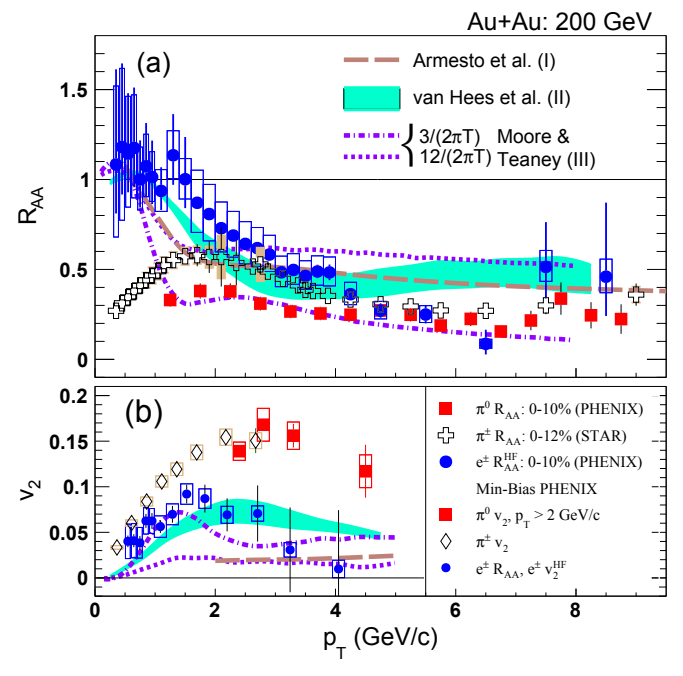

Figure 2. The nuclear modification factor, $\mathrm{R}_{\mathrm{AA}}^{\mathrm{HF}}$, for HF electrons compared with the $\mathrm{R}_{\mathrm{AA}}$ of $\pi^{0}$ in central $\mathrm{Au}+\mathrm{Au}$ collisions at $\sqrt{s_{N N}}=200 \mathrm{GeV}$, see panel (a). Panel (b) considers the anisotropic flow of HF electrons $\mathrm{v}_{2}^{H F}$ with that of $\mathrm{v}_{2}$ of $\pi^{0}$ and $\pi^{ \pm}$in minimumbias $\mathrm{Au}+\mathrm{Au}$ collisions. For more details see Ref. [4]

nature of quark confinement in hadrons is crucial, and yet this remains a poorly understood aspect of the quark-gluon description of matter. Collision experiments of heavy nuclei at high energies provide the unique opportunity to create and study the properties of the QGP in the laboratory.

Hadrons carrying heavy quarks, i.e. charm $(c)$ or bottom $(b)$, are consider important probes of the hot and dense medium, QGP, created in relativistic heavy-ion collisions. Heavy quark-antiquark pairs are mainly produced in initial hard scattering processes of partons. While some of the produced pairs form bound quarkonia, the vast majority hadronize into open heavy flavor particles. They interact through the medium and are expected to be sensitive to its energy density through the mechanism of parton energy loss. Due to the large mass of heavy quarks the suppression of small-angle gluon radiation should reduce their energy loss, and consequently any suppression of heavy-quark mesons like $D$ and $B$ mesons at high- $p_{T}$ is expected to be smaller than that observed for hadrons consisting of light quarks [3]. We quantify the medium effects on high- $p_{\mathrm{T}}$ production in nucleus-nucleus collisions, $\mathrm{A}+\mathrm{A}$, with the nuclear modification factor which is defined as following:

$$
\mathrm{R}_{\mathrm{AA}}\left(\mathrm{p}_{\mathrm{T}}\right)=\frac{1}{\left\langle\mathrm{~N}_{\text {coll }}\right\rangle} \times \frac{\text { yield per } \mathrm{A}+\mathrm{A} \text { collision }}{\text { yield per } p+p \text { collision }}=\frac{1}{\left\langle\mathrm{~N}_{\mathrm{coll}}\right\rangle} \times \frac{\mathrm{d}^{2} \mathrm{~N}^{\mathrm{A}+\mathrm{A}} / \mathrm{dydp}_{\mathrm{T}}}{\mathrm{d}^{2} \mathrm{~N}^{p+p} / \mathrm{dydp}_{\mathrm{T}}}
$$

This factor reflects the deviation of measured distributions of nucleus-nucleus, $\mathrm{A}+\mathrm{A}$, transverse momentum, at given impact parameter $b$, from measured distributions of an incoherent superposition of nucleon-nucleon $(p+p)$ transverse momentum, scaled by the average number of expected binary collisions' $\left\langle N_{\text {coll }}\right\rangle$. This normalization often is known as "binary collisions scaling".

The rise of the nuclear modification factor with center-of-mass energy, from the SPS to RHIC and then to the LHC is presented in figure 1 . We observe that in the presented $p_{\mathrm{T}}$ region, charged 
hadron production at the LHC is found to be about $50 \%$ more suppressed than at RHIC, and has a similar suppression value as for neutral pions $\left(\pi^{0}\right)$ measured by PHENIX. These measurements of $R_{A A}\left(p_{\mathrm{T}}\right)$ at RHIC and LHC is consistent with a large energy loss in the medium causing it to become opaque to the propagation of high momentum partons $[4,5]$. In contrast, $R_{A A}\left(p_{\mathrm{T}}\right)$ for $\pi^{0}$ at SPS energy exhibit enhancement, as shown in figure 1. This effect of $R_{A A}\left(p_{\mathrm{T}}\right)$ suppression of the high- $p_{\mathrm{T}}$ hadron spectra at the mid-rapidity region in central $\mathrm{A}+\mathrm{A}$ collisions, proposed by Bjorken, Gyulassy, and others [6,7] rests on the expectation of a large energy loss of high momentum partons scattered in the initial stages of collisions in a medium with a high density of free color charges. According to the QCD theory, colored objects will lose energy via bremsstrahlung radiation of gluons. Such a mechanism strongly would degrade the energy of leading partons, as reflected in the reduced transverse momentum of leading particles in the jets emerging after their fragmentation into hadrons. This energy loss is expected to depend strongly on the color charge density of the created system, and the path length traversed by the propagating parton. It has been recognized that the elastic energy loss is too small to engender significant attenuation of the jet in the created medium [8]. Adding to this discovery of suppression of particles at high- $p_{\mathrm{T}}$, two very striking results were seen for open heavy flavor from the PHENIX experiment via the measurement of electrons from the semi-leptonic decays of hadrons carrying charm or bottom quarks. First, heavy mesons, despite their large mass, exhibit a suppression at high transverse momentum compared to that expected from $p+p$ collisions [4, 9-11]. This suppression is similar to that of light mesons, which implies a substantial energy loss of fast heavy quarks while traversing the medium, see figure 2(a). Second, an elliptic flow is observed for heavy mesons, electrons from semi-leptonic decays of hadrons carrying charm or bottom quarks, that is similar to that of light mesons like pions, as shown in figure 2(b).

\section{PHENIX Recent Heavy Flavor Results}

Heavy quark production has been studied by the PHENIX experiment via measurements of single leptons from semi-leptonic decays in both the electron channel at mid-rapidity and in the muon channel at forward-rapidity. Recently, the PHENIX Collaboration has installed a silicon vertex tracker both in central rapidity (VTX) and in forward rapidity (FVTX) regions, and has collected large data samples $[12,13]$. These two silicon trackers enhance the capability of heavy flavor measurements via precision tracking [14].

\subsection{Open Heavy Flavor - Single Electron/Muon Measurements}

The PHENIX experiment quantifies open heavy flavor production through the measurement of inclusive electrons or muons [15-18]. These analyses use a cocktail method to remove fake and real electrons/muons from the data sample. i.e. for the electron measurement, the electrons that come from either meson decay or photon conversions are measured and subtracted from the inclusive spectrum and the remainder is attributed to electrons $\left(e_{H F}^{ \pm}\right)$coming from the semi-leptonic decay of $D$ and $B$ mesons. The effects of CNM are expected to be present in the initial state of A + A collisions; however, this CNM enhancement is convolved with the suppressing effects of hot nuclear matter. Figure 3(a) shows $R_{d A u}\left(p_{\mathrm{T}}\right)$ and $R_{A A}\left(p_{\mathrm{T}}\right)$ for $e_{H F}^{ \pm}$and the neutral pion $\left(\pi^{0}\right)$, for which only small CNM effects are observed between $e_{H F}^{ \pm}$and $\pi^{0}$ for $p_{\mathrm{T}}<5 \mathrm{GeV} / \mathrm{c}$. Above $p_{\mathrm{T}} \approx 5 \mathrm{GeV} / \mathrm{c}$, where the CNM effects on both species, $e_{H F}^{ \pm}$and $\pi^{0}$, are small, their $R_{A A}\left(p_{\mathrm{T}}\right)$ values are consistent within uncertainties. However, in the range where CNM enhancement is large for $e_{H F}^{ \pm}$and small on $\pi^{0}$, the corresponding $e_{H F}^{ \pm} R_{A A}\left(p_{\mathrm{T}}\right)$ values are consistently above the $\pi^{0}$ values. This could suggest that the difference in the initial state cold nuclear matter effects due to the mass-dependent Cronin enhancement is reflected in the final 


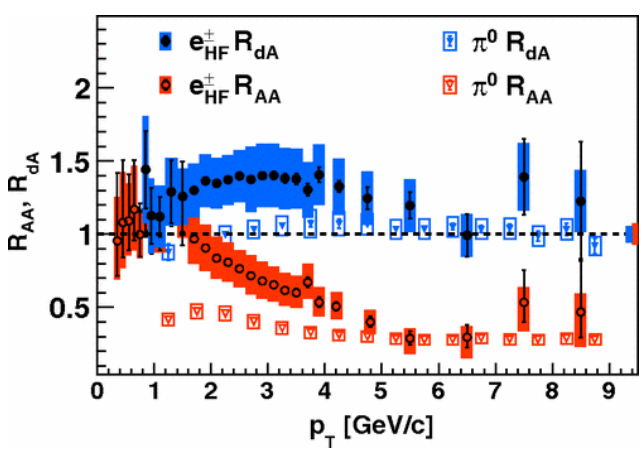

(a) $e_{H F}^{ \pm}$and $\pi^{0}: \mathrm{Au}+\mathrm{Au}$ vs. $d+\mathrm{Au}$ at $\sqrt{s_{N N}}=200 \mathrm{GeV}$

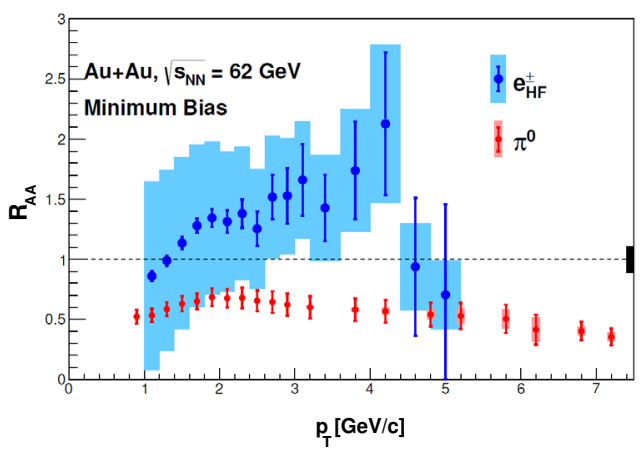

(b) $e_{H F}^{ \pm}$and $\pi^{0}: \mathrm{Cu}+\mathrm{Cu}$ at $\sqrt{s_{N N}}=62.4 \mathrm{GeV}$

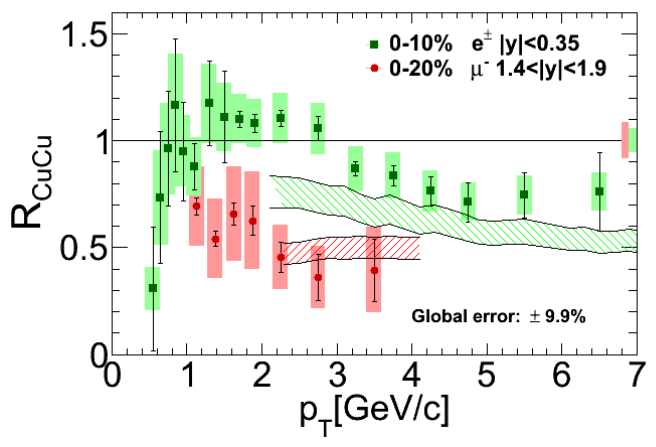

(c) $e_{H F}^{ \pm}$and $\mu_{H F}^{-}: \mathrm{Cu}+\mathrm{Cu}$ at $\sqrt{s_{N N}}=62.4 \mathrm{GeV}$

Figure 3. The nuclear modification factors $R_{d A u}\left(p_{\mathrm{T}}\right)$ and $R_{A A}\left(p_{\mathrm{T}}\right)$ for the $\pi^{0}, e_{H F}^{ \pm}$and $\mu_{H F}^{-}$: panels (a) and (b) for minimum-bias $d+\mathrm{Au}, \mathrm{Au}+\mathrm{Au}$ and panel (c) for central collisions $\mathrm{Cu}+\mathrm{Cu}[15,16]$.

state spectra of these particles in $\mathrm{Au}+\mathrm{Au}$ collisions, although alternate explanations involving massdependent partonic energy loss in the hot medium are not ruled out. To study the interplay between initial-state effects and final-states effects for heavy flavor $\left(e_{H F}^{ \pm}\right)$productions, PHENIX measured the $R_{A A}\left(p_{\mathrm{T}}\right)$ in $\mathrm{Au}+\mathrm{Au}$ collisions at low beam energy, $\sqrt{s_{N N}}=62.4 \mathrm{GeV}$. These $R_{A A}\left(p_{\mathrm{T}}\right)$ values for $e_{H F}^{ \pm}$ in $\mathrm{Au}+\mathrm{Au}$ collisions at $62.4 \mathrm{GeV}$ are compared to $\pi^{0} R_{A A}\left(p_{\mathrm{T}}\right)$ at the same energy as shown in figure $3(\mathrm{~b})$. At $62.4 \mathrm{GeV}$, the competition, if present, favors heavy flavor enhancement over suppression. This is consistent with previous results with hadrons where the Cronin enhancement increases as the collision energy decreases [19]. PHENIX measurements of heavy flavor muons $\left(\mu_{H F}^{-}\right)$at forward rapidity $(1.4<\mathrm{y}<1.9)$ in central $\mathrm{Cu}+\mathrm{Cu}$ collisions at $\sqrt{s_{N N}}=62.4 \mathrm{GeV}$ show a significant suppression [19]. The magnitude of this suppression at forward-rapidity in $\mathrm{Cu}+\mathrm{Cu}$ (shown in figure 3(c)) is compared to the suppression of $e_{H F}^{ \pm}$in central $\mathrm{Au}+\mathrm{Au}$ collisions at mid-rapidity at the same energy. As open heavy flavor is significantly more suppressed at forward-rapidity than at mid-rapidity in $\mathrm{Cu}+\mathrm{Cu}$, additional nuclear effects, such as gluon shadowing at low-Björken- $x$ or partonic energy loss in the nucleus, may be significant. The heavy flavor $e_{H F}^{ \pm}$and $\mu_{H F}^{-}$are compared in figure 3(c) to a theoretical prediction that combines the effects of partonic energy loss, energy loss from fragmentation and dissociation, and includes nuclear matter effects such as shadowing and Cronin enhancement due to parton scattering in the nucleus [20]. While consistent within uncertainties, the model predicts more suppression for heavy flavor electrons than seen in the data. 


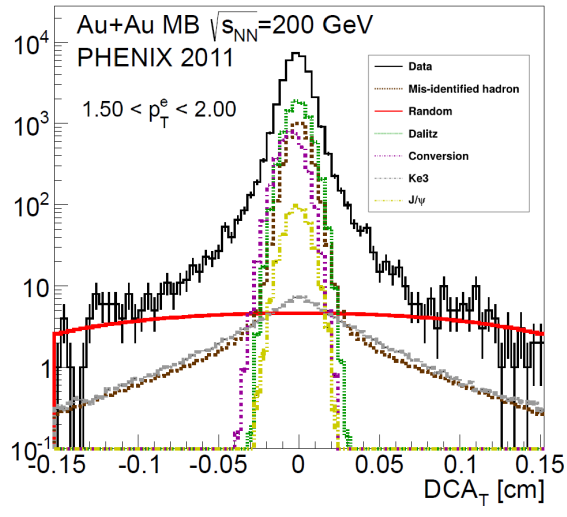

Figure 4. The $\mathrm{DCA}_{T}$ distribution for electrons with $1.5<p_{\mathrm{T}}^{e}<2.0 \mathrm{GeV} / \mathrm{c}$ in $\mathrm{Au}+\mathrm{Au}$ minimum-bias collisions at $\sqrt{s_{N N}}=200 \mathrm{GeV}$. Also shown are the normalized contributions for the various background components detailed in Ref. [22].

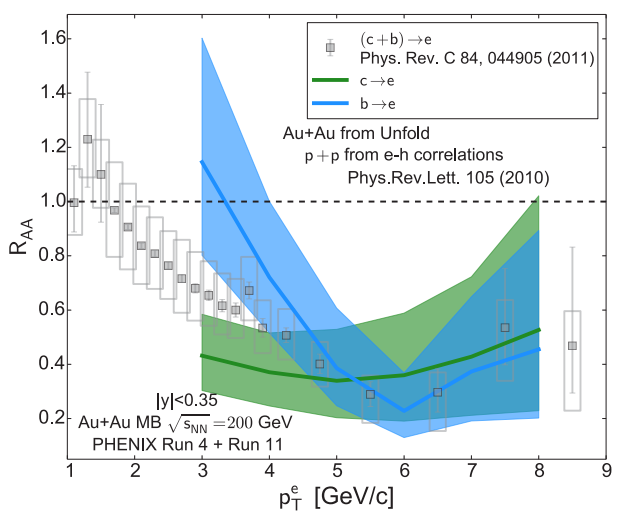

Figure 5. The nuclear modification factors $R_{\mathrm{AA}}$ of electrons from charm $(c \rightarrow e)$, bottom $(b \rightarrow e)$ decays and combined heavy flavor as a function of $p_{\mathrm{T}}^{e}$ in $\mathrm{Au}+\mathrm{Au}$ minimum-bias collisions at $\sqrt{s_{N N}}=200 \mathrm{GeV}$. For more details see Ref. [22].

The large suppression $R_{\mathrm{AA}}$ of $e_{H F}^{ \pm}$at high- $p_{\mathrm{T}}$ in $\mathrm{Au}+\mathrm{Au}$ collisions at $\sqrt{s_{N N}}=200 \mathrm{GeV}$, shown in figures 2 and 3, and similar to that of light mesons $\pi^{0}$, is the more striking results because perturbative QCD calculations indicate a substantial contribution from bottom quark decays for $p_{\mathrm{T}}>5 \mathrm{GeV} / \mathrm{c}$ [21]. For the specific purpose of separating the contributions of charm and bottom quarks at mid-rapidity, the PHENIX collaboration has added micro-vertexing capabilities in the form of a silicon vertex tracker (VTX) $[12,13]$. The different lifetimes and kinematics for charm and bottom hadrons decaying to electrons enable separation of their contributions with measurements of displaced tracks [14, 22]. Figure 4 shows the $\mathrm{DCA}_{T}$ distribution (in transverse plane) for electrons $\left(e^{ \pm}\right)$in $1.5<p_{\mathrm{T}}^{e}<2.0 \mathrm{GeV} / \mathrm{c}$ range obtained in $\mathrm{Au}+\mathrm{Au}$ minimum-bias collisions at $\sqrt{s_{N N}}=200 \mathrm{GeV}$ using data set of RHIC Run 2011. In order to extract the heavy flavor contributions, all background components, as shown in figure 4 , were fully accounted for and their $\mathrm{DCA}_{\mathrm{T}}$ shapes as a function of $p_{\mathrm{T}}$ incorporated. The nuclear modifications, $R_{A A}^{c \rightarrow e}$, and $R_{A A}^{b \rightarrow e}$, for charm and bottom hadron decays respectively are presented in figure 5 and detailed in Ref [22].

\section{$2.2 \psi(1 S)$ and $\psi(2 S)$ Mesons Production Versus System Size}

Figure 6 presents the measured di-muon mass spectra from $p+p, p+\mathrm{Al}, p+\mathrm{Au}$, and ${ }^{3} \mathrm{He}+\mathrm{Au}$ collisions at $\sqrt{s_{N N}}=200 \mathrm{GeV}$. These distributions comprise peaks at the $\psi(1 S)$ and $\psi(2 S)$ masses on top of correlated background from charm and bottom hadron decays and Drell-Yan pairs, plus combinatorial background from light meson decays and hadrons, which are not stopped in the absorbers [24]. From figure 6 it is apparent that the $\psi(2 S)$ peaks are suppressed relative to the $\psi(1 S)$ peak in the columns on the left (in the A-going direction). Quantitative comparisons are accomplished by calculating the double ratio of $\psi(2 S) / \psi(1 S)$ production in $\mathrm{p} / \mathrm{He}+\mathrm{A}$ collisions to the ratio found in $p+p$ collisions, as shown in figure 7. At forward rapidity, the double ratio is consistent with unity in all three collision systems, indicating that any possible nuclear effects on the two charmonium states are comparable. Because these states are not fully formed until after they exit the nucleus, the fact that any nuclear effects have an equal magnitude on both states suggests that there are no significant final 

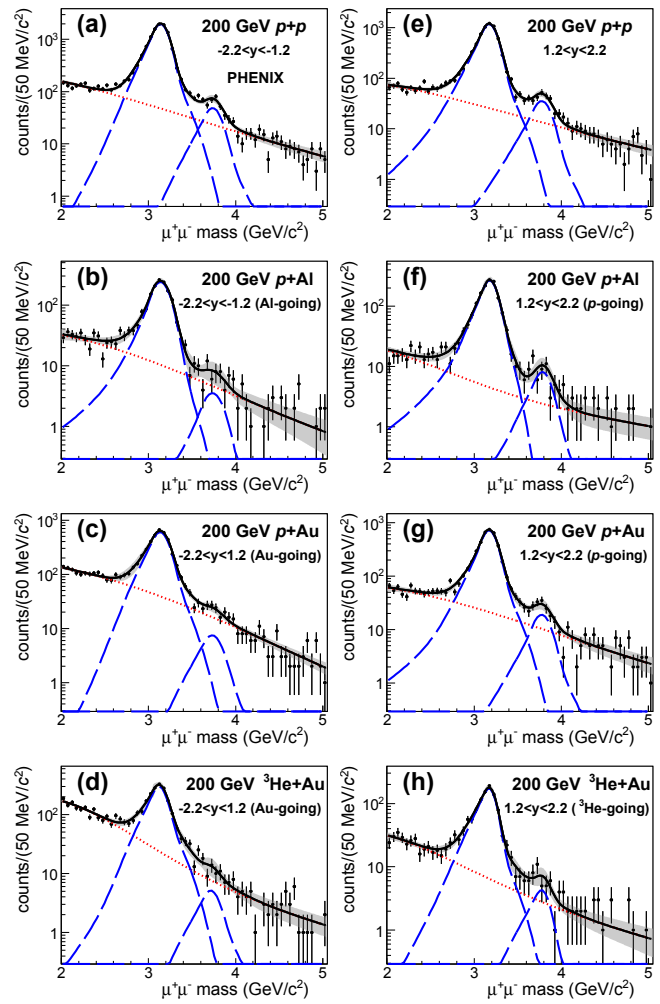

Figure 6. The measured di-muon mass spectra with fits from the $(\mathrm{a}-\mathrm{d})$ South and $(\mathrm{e}-\mathrm{h})$ North PHENIX muon arms, for $p+p, p+\mathrm{Al}, p+\mathrm{Au}$, and ${ }^{3} \mathrm{He}+\mathrm{Au}$ collisions at $\sqrt{s_{N N}}=200 \mathrm{GeV}$. The total fit is the solid [black] line with a shaded [gray] band representing the $90 \%$ confidence level. The dashed [blue] and dotted [red] lines represent the contributions from the resonances and background, respectively. For more details see Ref. [24].

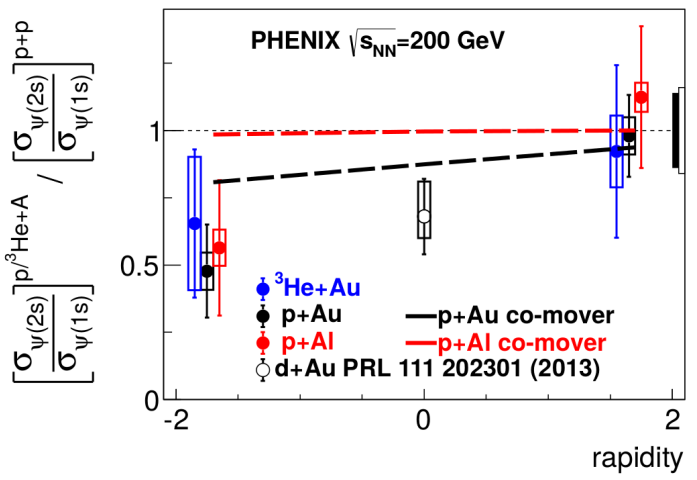

Figure 7. The double ratio of $\psi(2 S) / \psi(1 S)$ mesons measured in in $p+\mathrm{Al}, p+\mathrm{Au}$, and ${ }^{3} \mathrm{He}+\mathrm{Au}$ collisions to that same ratio in $p+p$ collisions at $\sqrt{s_{N N}}=200 \mathrm{GeV}$, with a calculation based on breakup by comoving particles. The bars (boxes) on the data points represent the statistical (systematic) uncertainties, and the shaded (open) box around unity represents the global uncertainty on the forward/backward (mid) rapidity data. For more details see Ref. [24].

state effects on the pair which occur in this rapidity region. At backward rapidity, the ratios in all collision systems are suppressed by a factor of two. The mechanism for this preferential suppression of the $\psi(2 S)$ relative to the $\psi(1 S)$ is expected to occur after the $\psi(1 S)$ formation time. A significant difference in the late stages of the collision between this region and forward rapidity suggests the presence of a larger number of comoving hadrons [24].

\section{STAR Recent Heavy Flavor Results}

The study of heavy flavor in relativistic nuclear collisions follows two different approaches: (1) The direct reconstruction of the heavy flavor meson, and (2) the identification of electrons and muons from semi-leptonic decays of such mesons. However, the rare production of heavy quarks and large combinatorial background in heavy-ion collisions makes the measurements of heavy flavor particles 


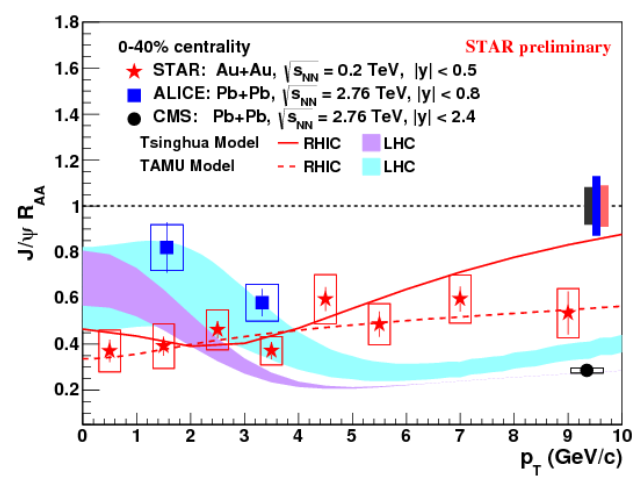

Figure 8. The nuclear modification factors $R_{\mathrm{AA}}$ as a function of $p_{\mathrm{T}}$ at RHIC (red star) and at LHC (blue square, black circle). The lines and bands indicate transport model calculations for RHIC and LHC energies [25].

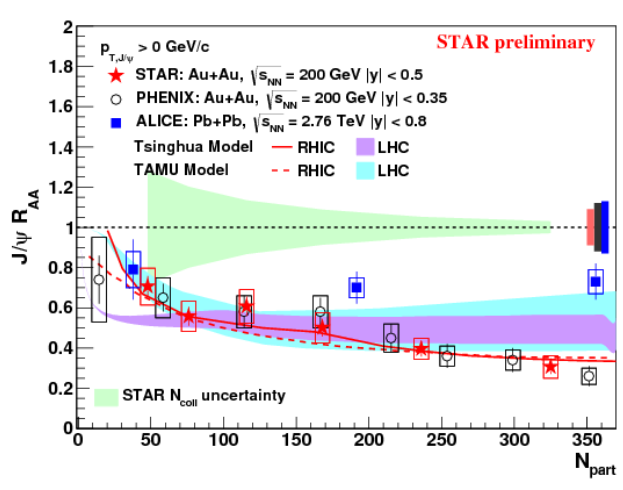

Figure 9. The nuclear modification factors $R_{\mathrm{AA}}$ for integrated $p_{\mathrm{T}}$ as a function of $\mathrm{N}_{\text {part }}$ in $\mathrm{Au}+\mathrm{Au}$ and $\mathrm{Pb}+\mathrm{Pb}$ collisions at RHIC and LHC energies respectively [25].

very difficult with the STAR detectors. To overcome the challenge of the combinatorial background, the STAR Collaboration upgraded the detector by adding the heavy flavor tracker (HFT) and the muon telescope detector (MTD) in 2014.

\section{1 $J / \psi$ and $\Upsilon$ Measurements in $\mathrm{Au}+$ Au collisions at Mid-rapidity}

Figure 8 presents the nuclear modification factor of $J / \psi R_{\mathrm{AA}}$ measurements in the di-muon decay channel in $0-40 \%$ central $\mathrm{Au}+\mathrm{Au}$ collisions at $\sqrt{s_{N N}}=200 \mathrm{GeV}$ at mid-rapidity region, compared with LHC results [25-27]. We observe a strong suppression at RHIC at low $p_{\mathrm{T}}$ indicating that dissociation plays a significant role in this $p_{\mathrm{T}}$ range. The increasing trend of $R_{\mathrm{AA}}$ at RHIC at high- $p_{\mathrm{T}}$ region could be interpreted by formation-time effects and feed-down of $B$ hadrons. The least suppression of $J / \psi$ at LHC at low- $p_{\mathrm{T}}$ indicates larger regeneration contribution due to higher charm cross section, while more suppression of $J / \psi$ at LHC at high- $p_{\mathrm{T}}$ indicates larger dissociation rate due to higher temperature of the medium. Transport Models [28-31], including dissociation and regeneration effects, describe qualitatively the $R_{\mathrm{AA}}$ of $J / \psi$ as a function of $p_{\mathrm{T}}$ at RHIC and LHC given energies [25]. Figure 9 presents the $J / \psi R_{\mathrm{AA}}$ centrality dependence for integrated $p_{\mathrm{T}}$. We observe that both models reproduce centrality dependence at RHIC, but aim to overestimate suppression (or underestimate $R_{\mathrm{AA}}$ of $J / \psi)$ at LHC. The di-muon mass spectrum in $\Upsilon$ state mass range in $\mathrm{Au}+\mathrm{Au}$ collisions at $\sqrt{s_{N N}}=$ $200 \mathrm{GeV}$ is shown in figure 10 . We notice signs of an indication of $\Upsilon(2 S+3 S)$ signals in the di-muon decay channel at mid-rapidity region. Using simultaneous fit to the like-sign and unlike-sign distributions, the raw yields of $\Upsilon$ states are obtained [25]. The comparison of the fitted $\Upsilon(2 S+3 S) / \Upsilon(1 S)$ ratio with the world-wide $p+p$ data [32] and CMS data [33, 34] is shown in figure 11 .

\section{Conclusions}

Understanding the properties of strongly interacting matter at high temperatures has been a central goal of the STAR and PHENIX experiments at RHIC. The large suppression $R_{\mathrm{AA}}$ of electrons from 


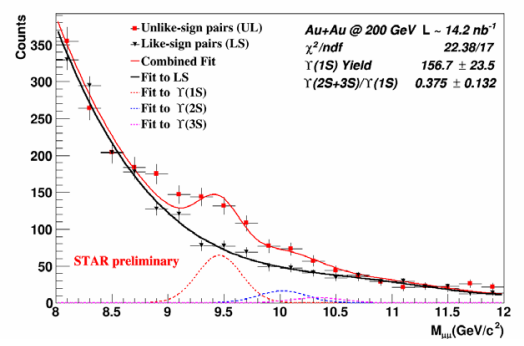

Figure 10. The di-muon mass spectrum in the $\Upsilon$ state mass range from $\mathrm{Au}+\mathrm{Au}$ collisions at $\sqrt{s_{N N}}=200 \mathrm{GeV}$ in mid-rapidity region [25].

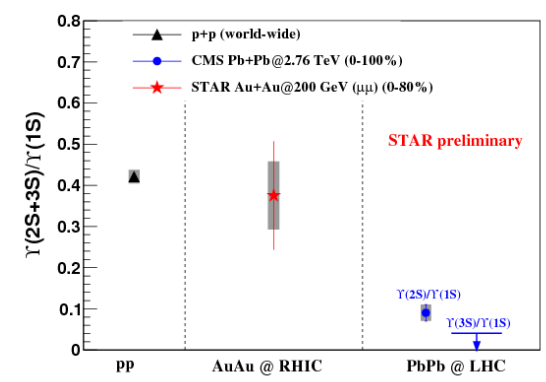

Figure 11. $\Upsilon(2 S+3 S) / \Upsilon(1 S)$ ratio for world-wide $p+p$ data, and for heavy-ion collisions at RHIC, and LHC energies [25].

the semi-leptonic decays of hadrons carrying charm or bottom quarks at high- $p_{\mathrm{T}}$ in $\mathrm{Au}+\mathrm{Au}$ collisions at $\sqrt{s_{N N}}=200 \mathrm{GeV}$, and similar to that of light mesons $\pi^{0}$, is the more striking results because perturbative QCD calculations indicate a substantial contribution from bottom quark decays for $p_{\mathrm{T}}>5 \mathrm{GeV} / \mathrm{c}$. The PHENIX experiments established measurements of $\psi(1 S)$ and $\psi(2 S)$ production as a function of system size, $p+p, p+\mathrm{Al}, p+\mathrm{Au}$, and ${ }^{3} \mathrm{He}+\mathrm{Au}$ collisions at $\sqrt{s_{N N}}=200 \mathrm{GeV}$. In $\mathrm{p} /{ }^{3} \mathrm{He}+\mathrm{A}$ collisions at forward rapidity, we observe no difference in the $\psi(2 S) / \psi(1 S)$ ratio relative to $p+p$ collisions. At backward rapidity, where the comoving particle density is higher, we find that the $\psi(2 S)$ is preferentially suppressed by a factor of two. STAR Collaboration presents the first $J / \psi$ and $\Upsilon$ measurements in $\mathrm{Au}+\mathrm{Au}$ collisions at $\sqrt{s_{N N}}=200 \mathrm{GeV}$ in the di-muon decay channel at midrapidity region. We observe a clear $J / \psi R_{\mathrm{AA}}$ suppression and qualitatively well described by transport models, including dissociation and regeneration simultaneously.

\section{References}

[1] E. Shuryak, Phys. Rep. 61, 71 (1980)

[2] L. McLerran, Rev. Mod. Phys. 58, 1021 (1986)

[3] Y. L. Dokshitzer and D. E. Kharzeev, Phys. Lett. B 519, 199 (2001)

[4] R. Nouicer, European Physical Journal Plus 131, 70 (2016)

[5] S. M. Pari et al., International Journal of Modern Physics E, 25, No. 6, 1650040 (2016)

[6] J. D. Bjorken, Phys. Rev. D 27, 140 (1983)

[7] M. Gyulassy et al. Phys. Lett. B 243, 432 (1990)

[8] M. H. Thoma et al., Nucl. Phys. B 351, 491 (1991)

[9] S.S. Adler et al. (PHENIX Collaboration), Phys. Rev. Lett. 98, 172302 (2007)

[10] S.S. Alder et al. (PHENIX Collaboration), Phys. Rev. Lett. 96, 032301 (2006)

[11] J. Adams et al. (STAR Collaboration), Phys. Rev. Lett. 94, 062301 (2005)

[12] R. Nouicer et al., Journal of Instrumentation (JINST), 4, P04011 (2009)

[13] R. Nouicer et al., Nuclear Instruments and Methods in Physics Research B 261, 10671071 (2007)

[14] R. Nouicer et al. (PHENIX Collaboration), Nuclear Physics A 904-905C, 647 (2013)

[15] R. Nouicer et al. (PHENIX Collaboration), Nuclear Physics A 862, 64 (2011)

[16] R. Nouicer et al. (PHENIX Collaboration), Journal of Physics : Conf. Series 420, 012021 (2013) 
[17] A. Adare et al. (PHENIX Collaboration), Phys. Rev. Lett. 109, 242301 (2012)

[18] A. Adare et al. (PHENIX Collaboration), Phys. Rev. Lett. 112, 252301 (2014)

[19] A. Adare et al. (PHENIX Collaboration), Phys. Rev. C 86, 024909 (2012)

[20] R. Sharma et al., Phys. Rev. C 80, 054902 (2009)

[21] M. Cacciari et al., Phys. Rev. Lett. 95, 122001 (2005)

[22] A. Adare et al. (PHENIX Collaboration), Phys. Rev. Lett. 93, 034904 (2016)

[23] H. Satz, Journal of Physics G 32, R25 (2006)

[24] A. Adare et al. (PHENIX Collaboration), arXiv:1609.06550 [nucl-ex]

[25] T. Todoroki et al. (STAR Collaboration), arXiv:1612.02499

[26] B. Abelev et al. (ALICE Collaboration), Phys. Let. B 734, 314 (2014)

[27] S. Chatrchyan et al. (CMS Collaboration), JHEP 05, 063 (2012)

[28] Y. Liu et al., Phys. Let. B 678, 72 (2009)

[29] K. Zhou et al., Phys. Rev. C89, 054911 (2014)

[30] X. Zhao et al., Phys. Rev. C 82, 064905 (2010)

[31] X. Zhao et al., Nucl. Phys. A 859, 114 (2011)

[32] W. Zha et al., Phys. Rev. C 88, 067901 (2013)

[33] S. Chatrchyan et al. (CMS Collaboration), Phys. Rev. Lett. 109, 222301 (2012)

[34] S. Chatrchyan et al. (CMS Collaboration), JHEP 04, 103 (2014) 